\title{
Parity nonconservation in deuteron photoreactions
}

\author{
T. M. Partanen $*$ and J. A. Niskanen \\ Department of Physical Sciences, P. O. Box 64, FIN-00014 University of Helsinki, Finland
}

\begin{abstract}
We calculate the asymmetries in parity nonconserving deuteron photodisintegration due to circularly polarized photons $\vec{\gamma} d \rightarrow n p$ with the photon laboratory energy ranging from the threshold up to $10 \mathrm{MeV}$ and the radiative capture of thermal polarized neutrons by protons $\vec{n} p \rightarrow \gamma d$. We use the leading order electromagnetic Hamiltonian neglecting the smaller nuclear exchange currents. Comparative calculations are done by using the Reid93 and Argonne $v_{18}$ potentials for the strong interaction and the DDH and FCDH "best" values for the weak couplings in a weak one-meson exchange potential. A weak $N \Delta$ transition potential is used to incorporate also the $\Delta(1232)$-isobar excitation in the coupled-channels formalism.
\end{abstract}

\section{INTRODUCTION}

Due to the incessant presence of the strong interaction, the hadronic weak interaction in the quark flavour conserving sector of the Standard Model is not completely understood. Even though the weak interaction between quarks is well known at high energies, its properties are hard to extract in the nonperturbative regime of quantum chromodynamics (QCD). This is due to the complicated structure of nuclear systems along with coinciding dynamics of QCD. It seems an almost hopeless challenge to distinguish directly about the size of seven orders of magnitude smaller weak interaction effects from those of QCD. Fortunately, the weak interaction leaves a unique signature in the form of parity nonconservation (PNC), which provides a tiny but non-vanishing observable.

For the past three decades the PNC calculations between nucleons have been based on the use of the DDH [1] potential. Unfortunately, the potential requires the knowledge of several weak meson-nucleon coupling constants, which are still to date inadequately known. Today's contemporary attempts to determine PNC amplitudes more and more often harness QCD based chiral perturbation theory $(\chi \mathrm{PT})$. Unlike the phenomenological meson-exchange model, $\chi \mathrm{PT}$ provides a systematic and model-independent way to study hadronic reactions at low energies. The updated $\chi \mathrm{PT}$ based approach leads to a systematic expansion of PNC amplitudes in terms of low-energy constants (LECs) which have a straightforward correspondence with the weak DDH meson-nucleon couplings, see e.g. refs. [2, 3].

Out of all the possible candidates for asymmetry and polarization observables, in this paper we focus on the asymmetries associated with PNC deuteron photodisintegration by circularly polarized photons $\vec{\gamma} d \rightarrow n p$ and thermal polarized neutron capture $\vec{n} p \rightarrow \gamma d$. It is further to be noted that the photon polarization of $n p \rightarrow \vec{\gamma} d$ at threshold equals the photon asymmetry of the time-reversed reaction $\vec{\gamma} d \rightarrow n p$ at threshold. The threshold behaviour of the asymmetry/polarization in reactions $\vec{\gamma} d \leftrightarrow n p$ can be shown to be insensitive to the $\pi$-meson exchange, which represents the long-range $\Delta I=1$ part of the PNC interaction. Therefore, the threshold region is essentially dominated by the exchanges of heavy mesons $(\rho, \omega)$ and thus also the relatively long-ranged $\Delta$-excitation could occur more clearly highlighted than what it would if it appeared in a background where the pion is more intensely present. To our knowledge this effect has only been checked in the form of exchange currents in refs. [4, [5]. Contrary to the reactions $\vec{\gamma} d \leftrightarrow n p$ at the threshold, the PNC $\pi$-exchange is predominant in the low-energy reaction $\vec{n} p \rightarrow \gamma d$.

There are various theoretical works on the PNC reactions $\vec{\gamma} d \rightarrow n p$ [4, 6- 12], $n p \rightarrow \vec{\gamma} d$ [12 19], and $\vec{n} p \rightarrow \gamma d$ [4, 5, 12, 15, 17, 18, 20 28]. The calculations are typically carried out exploiting the old meson-exchange picture, aside from some of the recent works, which apply the modern state-of-art techniques, e.g. such as the pionless effective field theory $\mathrm{EFT}(\pi)$ and heavy-baryon chiral perturbation theory $\mathrm{HB} \chi \mathrm{PT}$. The results more or less agree in the threshold region. The energy regime of several $\mathrm{MeV}$ above the threshold of the deuteron photodisintegration is investigated in refs. [4, 7 -10]. Again, in that regime, the results are similar except in ref. [7], which differs by an exceptionally large pion contribution. The difference is discussed in detail in ref. [10]. Up to date, for the reaction $\vec{\gamma} d \rightarrow n p$, there exist only two experimental data points: $(7.7 \pm 5.3) \times 10^{-6}$ and $(2.7 \pm 2.8) \times 10^{-6}$ at the photon energies of 3.2 and $4.1 \mathrm{MeV}$ respectively [29]. The latest photon polarization measurement for the inverse reaction $n p \rightarrow \vec{\gamma} d$ gives the value of $(1.8 \pm 1.8) \times 10^{-7}[30]$. The data from the 1980's for both the reactions are consistent

\footnotetext{
*Electronic address: tero.partanen@helsinki.fi

${ }^{\dagger}$ Electronic address: jouni.niskanen@helsinki.fi
} 
with zero with rather a poor precision and, therefore, new and more accurate experimental data, e.g. 31, 32], would be appreciated.

The asymmetry of the reaction $\vec{n} p \rightarrow \gamma d$ was also measured previously in the 70's and 80's, resulting in the insufficiently accurate values of $(0.6 \pm 2.1) \times 10^{-7}[33]$ and $-(1.5 \pm 4.8) \times 10^{-8}[34]$. However, there is currently an ongoing experiment (NPDGamma) on the reaction $\vec{n} p \rightarrow \gamma d$ with cold neutrons at LANSCE and SNS with a preliminary result, which sets the asymmetry within the limits of $(-1.2 \pm 2.1$ (stat.) $\pm 0.1($ sys. $)) \times 10^{-7}[35]$. The oncoming measurements of the NPDGamma experiment aim to improve the accuracy up to a level of $20 \%$ of the typical theoretical prediction $-5 \times 10^{-8}$, which employs the DDH "best value" for the weak $\pi N N$ coupling constant $h_{\pi}^{(1)}$. The asymmetry is straightforwardly proportional to the $h_{\pi}^{(1)}$ and therefore the current experiment is about to shed some light on the uncertain value of the coupling.

In this paper we study PNC in the above reactions using two modern phenomenological strong potentials and post-DDH weak couplings. Furthermore, we want to estimate the size of the aforementioned $\Delta$ effect, which was found to be significant in PNC elastic $\overrightarrow{p p}$ scattering at higher energies [36, 37]. As in these works our calculation is carried out within the framework of the coupled channels meson-exchange model and hence, according to the common practice, we utilize the DDH potential [1] as the starting point for the PNC $\pi-, \omega-$, and $\rho$-exchanges extending to use the weak couplings of ref. 38] consistent with the presence of the $\Delta$. We take account of exchange currents only in the extent they exist when the Siegert's theorem [39] is applied. They have been considered more explicitly e.g. in refs. [4, 5, 10]. In practise we use the electromagnetic Hamiltonian under the dipole approximation, which allows the PNC deuteron breakup to have four $p n$ continuum channels ${ }^{1} S_{0},{ }^{3} P_{0},{ }^{3} P_{1}$, and ${ }^{3} P_{2}-{ }^{3} F_{2}$ in the expansion up to $P$ waves.

This paper is organized as follows. In sect. II we give the appropriate forms of the electromagnetic Hamiltonian, the scattering and deuteron wavefunctions, and the PNC one-meson exchange $N N$ and $N \Delta$-transition potentials. The forms of the spin observables $\mathcal{A}_{\vec{\gamma}}$ and $\mathcal{A}_{\vec{n}}$ respectively of the reactions $\vec{\gamma} d \rightarrow n p$ and $\vec{n} p \rightarrow \gamma d$ are also given. Summary of the results and conclusions are presented in sect. III.

\section{THEORY}

\section{A. Interactions}

The observable asymmetries in the photoreactions arise from an interference between the strong and weak interactions giving rise to simultaneous presence of the photomagnetic and photoelectric effects. The interference appears when these two parallel processes share the same final state quantum numbers, and the PNC observables are obtained in terms of products of PC and PNC partial wave amplitudes. This is illustrated in fig. 11, where the mechanisms $(N N, N \Delta$ with PC and PNC forces) are shown alongside with the quantum numbers of the possible states up to $P$-wave final nucleons. The electromagnetic perturbing Hamiltonian $\hat{H}_{\mathrm{e} . \mathrm{m} .}=\hat{H}_{\mathrm{E} 1}^{N N}+\hat{H}_{\mathrm{M} 1}^{N N}+\hat{H}_{\mathrm{M} 1}^{N \Delta}$ which takes care of the disintegration and formation of the deuteron is considered in the dipole approximation $e^{ \pm i \boldsymbol{k}_{\gamma} \cdot \boldsymbol{r}} \approx 1$.

The Hamiltonian for absorption of a photon may be written as

$$
\hat{H}_{\mathrm{e} . \mathrm{m} .}^{\lambda}=-i \sqrt{\frac{\alpha \pi \omega_{\gamma}}{2}} \hat{\boldsymbol{\epsilon}}_{\boldsymbol{k}_{\gamma} \lambda} \cdot \sum_{i}\left[\left(1+\hat{\tau}_{i}^{z}\right) \boldsymbol{r}_{i}+\frac{1}{2 M}\left(\left(1+\hat{\tau}_{i}^{z}\right) \boldsymbol{\ell}_{i}+\left(\mu_{s}+\mu_{v} \hat{\tau}_{i}^{z}\right) \boldsymbol{\sigma}_{i}+2 \mu^{\star}\left(\hat{T}_{i}^{z} \boldsymbol{S}_{i}+\text { h.c. }\right)\right) \times \hat{\boldsymbol{k}}_{\gamma}\right],
$$

where $\hat{\boldsymbol{\epsilon}}_{\boldsymbol{k}_{\gamma} \lambda}(\lambda= \pm 1)$ is the circular polarization vector of the incoming photon, $\omega_{\gamma}$ the center-of-mass energy of the photon, $\mu_{s}=0.88$ and $\mu_{v}=4.71$ the isoscalar and isovector magnetic moments of nucleons, $\alpha=e^{2}$ the fine-structure constant, and $M=939 \mathrm{MeV}$ the average nucleon mass. The $\gamma N \Delta$-vertex can occur in the presence of the M1 and E2 transitions. We consider only the dominant M1 multipole and neglect the small E2 effect. The value of the transition magnetic moment is given by the quark model as $\mu^{\star}=f^{\star} \mu_{v} / 2 f=3 \sqrt{2} \mu_{v} / 5$ [40]. The nucleon-Delta spin and isospin transition operators are denoted as $\boldsymbol{S}$ and $\hat{T}_{z}[41]$.

In the presence of possible channel coupling the final state scattering wavefunctions are of the form

$$
\psi_{S M_{S}}^{T(-)}(\boldsymbol{k}, \boldsymbol{r})=\frac{4 \pi \sqrt{2}}{k r} \sum_{\kappa^{\prime} J M} \sum_{L M_{L}} i^{L}\left\langle L M_{L} S M_{S} \mid J M\right\rangle Y_{L M_{L}}^{*}(\hat{\boldsymbol{k}}) \mathcal{U}_{\kappa \kappa^{\prime}}^{J(-)}(k, r) \mathcal{Y}_{J M}^{L^{\prime} S^{\prime}}(\hat{\boldsymbol{r}})\left|T^{\prime} 0\right\rangle
$$

where the superscript " $(-)$ " on the wavefunctions refers to the incoming wave boundary conditions. The $|T 0\rangle$ are the relevant isospin states for the $p n$-interaction, $\mathcal{Y}_{J M}^{L S}(\hat{\boldsymbol{r}})$ the eigenfunctions of the coupled total angular momentum, and $\mathcal{U}_{\kappa \kappa^{\prime}}^{J(-)}(k, r)$ the complex-valued radial wavefunctions. The quantum numbers $L S T$ (relative orbital angular momentum $L$, total spin $S$, and total isospin $T$ ), which may be changed by the nuclear forces, are denoted for brevity by $\kappa$ which 


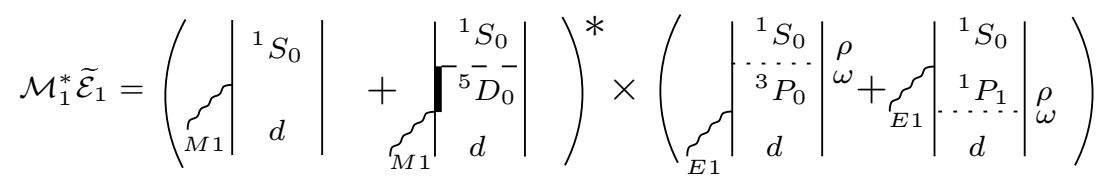





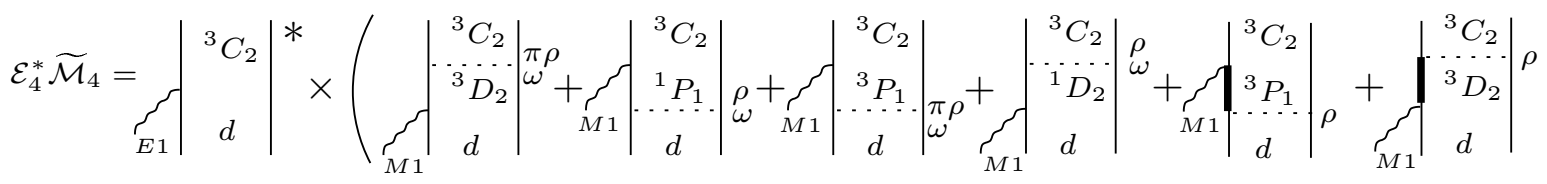

$$
\begin{aligned}
& +r_{M 1}\left|\begin{array}{c}
{ }^{3} C_{2} \\
{ }^{5} P_{1} \\
d
\end{array}\right| \rho+\underbrace{}_{M 1}\left|\begin{array}{c}
{ }^{3} C_{2} \\
{ }^{5} S_{2} \\
d
\end{array}\right| \rho \mid
\end{aligned}
$$

FIG. 1: Graphical representation for the $n p$ and the direct $N \Delta$ contributions in the deuteron photodisintegration. The dashed line represents the strong interaction, the dotted line the weak interaction, and the wavy line the incoming photon. For brevity the tensor coupled scattering states ${ }^{3} S_{1}-{ }^{3} D_{1}$ and ${ }^{3} P_{2}-{ }^{3} F_{2}$ are denoted by ${ }^{3} C_{1}$ and ${ }^{3} C_{2}$ respectively. Similarly, the $d$ stands for the standard PC deuteron ${ }^{3} S_{1}-{ }^{3} D_{1}$ state.

also refers to the main final wavefunction, whereas $\kappa^{\prime}$ labels the "small" component generated from it. The $J$ and $M$ relate to the total angular momentum and they are good quantum numbers under the nuclear forces. The calculated matrix elements ${ }^{(-)}\left\langle\boldsymbol{k} ; S M_{S} T\left|\hat{H}_{\mathrm{e} . \mathrm{m} .}^{\lambda}\right| M_{d}\right\rangle_{\mathcal{D}}$ will be expressed in terms of the wavefunctions with the outgoing boundary conditions " $(+)$ ", i.e. $\mathcal{U}_{\kappa \kappa^{\prime}}^{J(+)}(k, r)=\mathcal{U}_{\kappa \kappa^{\prime}}^{J(-) *}(k, r)$.

The deuteron wavefunction consists of a superposition of six relevant components: the standard PC $N N\left({ }^{3} S_{1},{ }^{3} D_{1}\right)$ and the tiny PNC $N N\left({ }^{1} P_{1},{ }^{3} P_{1}\right)$ and $N \Delta\left({ }^{3} P_{1},{ }^{5} P_{1}\right)$ states and may be written as

$$
\psi_{M_{d}}^{d}(\boldsymbol{r})=\sum_{\kappa_{d}} \frac{\mathcal{D}_{\kappa_{d}}(r)}{r} \mathcal{Y}_{1 M_{d}}^{L_{d} S_{d}}(\hat{\boldsymbol{r}})\left|T_{d} 0\right\rangle
$$

with the normalization $\int_{0}^{\infty} d r \sum_{i}^{6}\left|\mathcal{D}_{i}\right|^{2}=1$. The PC bound wavefunctions are real-valued and their PNC partners imaginary-valued. Both the scattering and bound state wavefunctions are obtained by solving coupled Schrödinger equations. The radial Schrödinger equation for the parity-admixed interaction reads

$$
\begin{aligned}
\left(\frac{\partial^{2}}{\partial r^{2}}\right. & \left.-\frac{L^{\prime}\left(L^{\prime}+1\right)}{r^{2}}+k^{2}\right) \mathcal{U}_{\kappa \kappa^{\prime}}^{J(+)}(k, r) \\
& =2 \mu \sum_{\kappa^{\prime \prime}} \int d \Omega_{\boldsymbol{r}} \mathcal{Y}_{L^{\prime \prime} S^{\prime \prime}}^{J M \dagger}(\hat{\boldsymbol{r}})\left\langle T^{\prime \prime} 0\left|\hat{V}^{\mathrm{PC}}(\boldsymbol{r})+\hat{V}^{\mathrm{PNC}}(\boldsymbol{r})\right| T^{\prime} 0\right\rangle \mathcal{Y}_{L^{\prime} S^{\prime}}^{J M}(\hat{\boldsymbol{r}}) \mathcal{U}_{\kappa \kappa^{\prime \prime}}^{J(+)}(k, r),
\end{aligned}
$$

where for $\hat{V}^{\mathrm{PC}}(\boldsymbol{r})$ the digonal $N N$ potential is taken as the phenomenological updated Reid soft core (Reid93) 42] and Argonne $v_{18}\left(\mathrm{~A} v_{18}\right)$ [43] potentials and respectively the $\Delta N$ as the mass difference $M_{\Delta}-M$ to generate the strong correlations. The other potentials are defined in eqs. (5) and (8)-(11). In the presence of $\Delta N$ channels the $N N$ potentials must be modified to avoid doubly counting the attraction from the channel coupling guaranteeing the phase equivalence. It is crucial for the result that it is done with high precision. The reduced masses $\mu$ for the initial $N N$ and $\Delta N$ states are respectively $\mu_{N N}=M / 2$ and $\mu_{N \Delta}=M M_{\Delta} /\left(M+M_{\Delta}\right)$. 
TABLE I: The weak $\alpha N N$ couplings $h_{\alpha}^{(i)}$ and $\alpha N \Delta$ couplings $h_{\alpha}^{\star(i)}$. The set of the first three weak couplings are the DDH "best values" [1] and the following five the FCDH "best values" [38]. The weak couplings are given in units of $10^{-7}$.

\begin{tabular}{c|ccc|ccccc|ccc}
\hline \hline & \multicolumn{9}{c}{ DDH } & \multicolumn{1}{c}{ FCDH } \\
& $h_{\alpha}^{(0)}$ & $h_{\alpha}^{(1)}$ & $h_{\alpha}^{(2)}$ & $h_{\alpha}^{(0)}$ & $h_{\alpha}^{(1)}$ & $h_{\alpha}^{(2)}$ & $h_{\alpha}^{\star(0)}$ & $h_{\alpha}^{\star(1)}$ & $g_{\alpha}$ & $\chi_{\alpha}$ & $\Lambda_{\alpha}(\mathrm{GeV})$ \\
\hline$\pi$ & - & 4.6 & - & - & 2.7 & - & - & - & 13.45 & - \\
$\rho$ & -11.4 & -0.2 & -9.5 & -3.8 & -0.4 & -6.8 & 7.6 & 7.6 & 2.79 & 3.71 \\
$\omega$ & -1.9 & -1.1 & - & -4.9 & -2.3 & - & - & 4.2 & 8.37 & -0.12 & 1.2 \\
\hline \hline
\end{tabular}

For the weak $N N$ interaction we use the DDH weak one-meson exchange nucleon-nucleon potential ref. [1]. In the case of $p n$-system, the relevant part of the potential is

$$
\begin{aligned}
\hat{V}_{N N}^{\mathrm{PNC}}(\boldsymbol{r})= & \frac{i h_{\pi}^{(1)} g_{\pi}}{2 \sqrt{2} M}\left(\boldsymbol{\tau}_{1} \times \boldsymbol{\tau}_{2}\right)_{z}\left(\boldsymbol{\sigma}_{1}+\boldsymbol{\sigma}_{2}\right) \cdot \hat{\mathcal{O}}_{\pi}^{-}-\frac{g_{\rho}}{M}\left[\left(h_{\rho}^{(0)} \boldsymbol{\tau}_{1} \cdot \boldsymbol{\tau}_{2}+\frac{h_{\rho}^{(2)}}{2}\left[\boldsymbol{\tau}_{1} \otimes \boldsymbol{\tau}_{2}\right]_{0}^{(2)}\right) \times\right. \\
& \left.\left(\left(\boldsymbol{\sigma}_{1}-\boldsymbol{\sigma}_{2}\right) \cdot \hat{\mathcal{O}}_{\rho}^{+}+i\left(1+\chi_{\rho}\right)\left(\boldsymbol{\sigma}_{1} \times \boldsymbol{\sigma}_{2}\right) \cdot \hat{\mathcal{O}}_{\rho}^{-}\right)-h_{\rho}^{(1)} \frac{\hat{\tau}_{1}^{z}-\hat{\tau}_{2}^{z}}{2}\left(\boldsymbol{\sigma}_{1}+\boldsymbol{\sigma}_{2}\right) \cdot \hat{\mathcal{O}}_{\rho}^{+}\right] \\
& -\frac{g_{\omega}}{M}\left[h_{\omega}^{(0)}\left(\left(\boldsymbol{\sigma}_{1}-\boldsymbol{\sigma}_{2}\right) \cdot \hat{\mathcal{O}}_{\omega}^{+}+i\left(1+\chi_{\omega}\right)\left(\boldsymbol{\sigma}_{1} \times \boldsymbol{\sigma}_{2}\right) \cdot \hat{\mathcal{O}}_{\omega}^{-}\right)+h_{\omega}^{(1)} \frac{\hat{\tau}_{1}^{z}-\hat{\tau}_{2}^{z}}{2}\left(\boldsymbol{\sigma}_{1}+\boldsymbol{\sigma}_{2}\right) \cdot \hat{\mathcal{O}}_{\omega}^{+}\right]
\end{aligned}
$$

where $\hat{\mathcal{O}}_{\alpha}^{-}=\left[-i \nabla, Y_{\alpha}(r)\right]$ and $\hat{\mathcal{O}}_{\alpha}^{+}=\left\{-i \nabla, Y_{\alpha}(r)\right\}$, with $\alpha=\pi, \rho, \omega$ are respectively the commutator and anticommutator in which the radial functions are

$$
Y_{\alpha}(r)=\frac{e^{-m_{\alpha} r}}{4 \pi r},
$$

if form factors are not used. In case monopole form factors of the type $\left(\Lambda_{\alpha}^{2}-m_{\alpha}^{2}\right) /\left(\boldsymbol{q}^{2}+m_{\alpha}^{2}\right)$, which we use here, are included in vertices, the modified Yukawa functions take the form

$$
Y_{\alpha}(r)=\frac{e^{-m_{\alpha} r}}{4 \pi r}-\frac{e^{-\Lambda_{\alpha} r}}{4 \pi}\left(\frac{1}{r}+\frac{\Lambda_{\alpha}^{2}-m_{\alpha}^{2}}{2 \Lambda_{\alpha}}\right) .
$$

In eq. (5) we have neglected the term proportional to $h_{\rho}^{(1)^{\prime}}$ because of the smallness and vagueness of the coupling and also the irrelevant $\propto\left(\hat{\tau}_{1}^{z}+\hat{\tau}_{2}^{z}\right)$ terms, which do not contribute in $p n$ interaction.

The PNC transition potential $(N N \leftrightarrow \Delta N)$ may be derived from the vertex interaction Hamiltonians (A1)-A8) of Appendix A, resulting in the potentials (8)-(10) for $\rho$-, $\omega$-, and $\pi$-exchanges respectively:

$$
\begin{aligned}
& V_{\rho N \Delta}^{\mathrm{PNC}}(\boldsymbol{r})=-\frac{1}{2 M}\left\{g_{\rho}\right. {\left[\left(h_{\rho}^{\star(0)}+\frac{h_{\rho}^{\star(1)}}{3}\right) \boldsymbol{T}_{1} \cdot \boldsymbol{\tau}_{2}+h_{\rho}^{\star(1)} \sqrt{\frac{2}{3}}\left[\boldsymbol{T}_{1} \otimes \boldsymbol{\tau}_{2}\right]_{0}^{(2)}\right] \boldsymbol{S}_{1} \cdot \hat{\mathcal{O}}_{\rho}^{+} } \\
&+ {\left[\left(g_{\rho}^{\star} h_{\rho}^{(0)}+g_{\rho}\left(h_{\rho}^{\star(0)}+\frac{h_{\rho}^{\star(1)}}{3}\right)\right) \boldsymbol{T}_{1} \cdot \boldsymbol{\tau}_{2}+\left(\frac{g_{\rho}^{\star} h_{\rho}^{(2)}}{2}+\sqrt{\frac{2}{3}} g_{\rho} h_{\rho}^{\star(1)}\right)\left[\boldsymbol{T}_{1} \otimes \boldsymbol{\tau}_{2}\right]_{0}^{(2)}\right.} \\
&\left.+h_{\rho}^{(1)} g_{\rho}^{\star} \hat{T}_{10}\right] i\left(1+\chi_{\rho}\right)\left(\boldsymbol{S}_{1} \times \boldsymbol{\sigma}_{2}\right) \cdot \hat{\mathcal{O}}_{\rho}^{-} \\
&- g_{\rho}\left[\left(h_{\rho}^{\star(0)}+\frac{h_{\rho}^{\star(1)}}{3}\right) \boldsymbol{\tau}_{1} \cdot \boldsymbol{T}_{2}+h_{\rho}^{\star(1)} \sqrt{\frac{2}{3}}\left[\boldsymbol{\tau}_{1} \otimes \boldsymbol{T}_{2}\right]_{0}^{(2)}\right] \boldsymbol{S}_{2} \cdot \hat{\mathcal{O}}_{\rho}^{+} \\
&+ {\left[\left(g_{\rho}^{\star} h_{\rho}^{(0)}+g_{\rho}\left(h_{\rho}^{\star(0)}+\frac{h_{\rho}^{\star(1)}}{3}\right)\right) \boldsymbol{\tau}_{1} \cdot \boldsymbol{T}_{2}+\left(\frac{g_{\rho}^{\star} h_{\rho}^{(2)}}{2}+\sqrt{\frac{2}{3}} g_{\rho} h_{\rho}^{\star(1)}\right)\left[\boldsymbol{\tau}_{1} \otimes \boldsymbol{T}_{2}\right]_{0}^{(2)}\right.} \\
&\left.\left.+h_{\rho}^{(1)} g_{\rho}^{\star} \hat{T}_{20}\right] i\left(1+\chi_{\rho}\right)\left(\boldsymbol{\sigma}_{1} \times \boldsymbol{S}_{2}\right) \cdot \hat{\mathcal{O}}_{\rho}^{-}\right\}+ \text {h.c. }, \\
& V_{\pi N \Delta}^{\mathrm{PNC}}(\boldsymbol{r})=i \frac{h_{\pi}^{(1)} g_{\pi}^{\star}}{2 \sqrt{2} M}\left(\left(\boldsymbol{T}_{1} \times \boldsymbol{\tau}_{2}\right)_{0} \boldsymbol{S}_{1}+\left(\boldsymbol{\tau}_{1} \times \boldsymbol{T}_{2}\right)_{0} \boldsymbol{S}_{2}\right) \cdot \hat{\mathcal{O}}_{\pi}^{-}+\mathrm{h} . c .,
\end{aligned}
$$




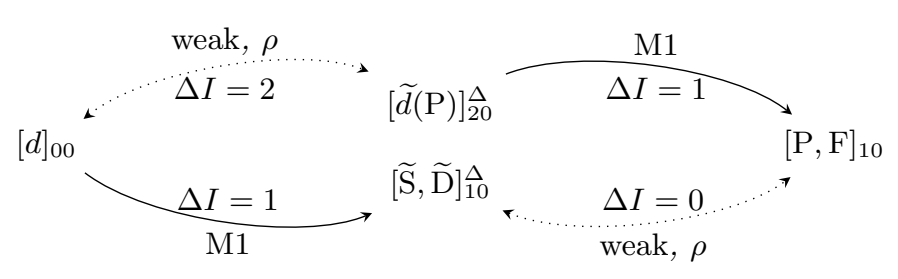

FIG. 2: Diagrammatic representation of the isospin change $\Delta I$ in the process of the PNC reaction $\vec{\gamma} d \rightarrow n p$ through the direct $\Delta$-channel. The subscript denotes the isospin state.

and

$$
\begin{aligned}
V_{\omega N \Delta}^{\mathrm{PNC}}(\boldsymbol{r})= & -\frac{g_{\omega} h_{\omega}^{\star(1)}}{2 M}\left[\left(\hat{T}_{10} \boldsymbol{S}_{1}-\hat{T}_{20} \boldsymbol{S}_{2}\right) \cdot \hat{\mathcal{O}}_{\omega}^{+}\right. \\
& \left.+i\left(1+\chi_{\omega}\right)\left(\hat{T}_{10}\left(\boldsymbol{S}_{1} \times \boldsymbol{\sigma}_{2}\right)+\hat{T}_{20}\left(\boldsymbol{\sigma}_{1} \times \boldsymbol{S}_{2}\right)\right) \cdot \hat{\mathcal{O}}_{\omega}^{-}\right]+ \text {h.c. }
\end{aligned}
$$

where $g_{\alpha}^{\star}=\sqrt{72 / 25} g_{\alpha}(\alpha=\pi, \rho)$ are the strong meson- $N \Delta$ couplings following from the quark model. It may be noted that in the case of pion-exchange the $\Delta$ is generated only at the strong vertex. The $\pi$ and $\rho$-mediated strong transition potential of the standard form [41] is

$$
\begin{aligned}
\hat{V}_{N \Delta}^{\pi, \rho}(\boldsymbol{r})= & \frac{g_{\pi} g_{\pi}^{\star}}{4 M^{2}} \boldsymbol{T}_{1} \cdot \boldsymbol{\tau}_{2}\left(\boldsymbol{S}_{1} \cdot \boldsymbol{\nabla}\right)\left(\boldsymbol{\sigma}_{2} \cdot \boldsymbol{\nabla}\right) Y_{\pi}(r) \\
& +\frac{g_{\rho} g_{\rho}^{\star}}{4 M^{2}}\left(1+\chi_{\rho}\right)^{2} \boldsymbol{T}_{1} \cdot \boldsymbol{\tau}_{2}\left(\boldsymbol{S}_{1} \times \boldsymbol{\nabla}\right)\left(\boldsymbol{\sigma}_{2} \times \boldsymbol{\nabla}\right) Y_{\rho}(r)+(1 \leftrightarrow 2)+\text { h.c. }
\end{aligned}
$$

involving a spin-spin and tensor part. Note that the strong $N \Delta$ transition potential (11) is more singular than $r^{-2}$ and thus necessarily requires regularization. Therefore, in order to be thoroughly consistent, we always use the modified Yukawa functions (7) in the presence of the $\Delta$.

The whole process of the PNC photodisintegration $\vec{\gamma} d \rightarrow n p$ is compelled to change the initial (PC bound) isosinglet state to the final (continuum) isovector state. The disintegration isospin transition operator allows only the $\Delta I=1$ transitions, with $\left\langle 10\left|\hat{T}_{i z}\right| 00\right\rangle=\left\langle 20\left|\hat{T}_{i z}\right| 10\right\rangle=\sqrt{2 / 3}$ being the only nonzero matrix elements, where $i=1,2$ labels the particle. Only the isovector mesons can couple to the PC $N \Delta$-vertex, which automatically excludes the $\omega$-exchange in such amplitudes. The weak $\pi$-exchange is also excluded, since the total isospin would not change in processes via $\Delta$ channels. For the same reason there is no contribution coming from the PNC $N \Delta \omega$ vertex. In general, all the structures of the PNC transition potential related to the isospin $\Delta I=1$ change are zero, and thus only the $\rho$-exchange occurs in the presence of the $\Delta$-channel, see fig. 2

The potential (8) has only one relevant isospin transition amplitude $1 \leftrightarrow 1$ in scattering and basically two $0 \leftrightarrow 0$ and $0 \leftrightarrow 2$ in the deuteron, from which only the latter one is nonzero. Following the usual practice in similar calculations, we also choose to use the DDH couplings in order to be comparable with the corresponding works. Unfortunately, there are no published weak $\alpha N \Delta(\alpha=\pi, \rho, \omega)$ couplings corresponding to the weak DDH $\alpha N N$ couplings. The "best" values of the weak $\alpha N \Delta$-couplings have been evaluated in newer analyses $[38$, 44$]$. To extract the pure $\Delta$ effect we shall use the FCDH values [38] for the needed weak $\alpha N \Delta$-couplings, even though they would not necessarily be entirely consistent with the other DDH couplings. However, we also study the effect of using the couplings from the consistent analysis of ref. [38].

It may be noted that, as a consequence of coupled-channel dynamics, in addition to the one-meson exchanges depicted in fig. 1 the $\Delta$ channels may also include possible higher order corrections, which are naturally taken into account as correlation effects. For instance, at the threshold, the leading $\Delta$ contributions originate from the onceiterated meson exchange diagrams presented in fig. 3, where eq. (11) is employed to take care of the strong $N \Delta$ transitions.

\section{B. Observables}

As a first test we calculate the total photon absorption cross-section $\sigma_{\gamma}=\sigma_{+}+\sigma_{-}$for the reaction $\gamma d \rightarrow n p$, obtained by Fermi's golden rule summing over the photon helicities and the final spin states and averaging over the 


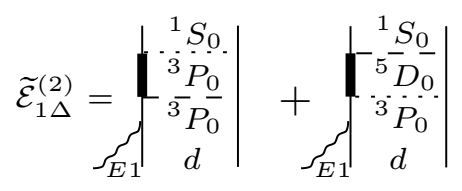

FIG. 3: Second order $\Delta$ corrections at the threshold.

two polarization directions of the photon and the three possible spin projections of the deuteron. Due to the identity of the final state particles (in the isospin formalism) the total cross section is also divided by two. Thus we have

$$
\sigma_{\gamma}=\frac{2 M}{9 k} \sum_{\kappa_{d} \kappa J}\left(\left|\mathcal{E}_{\kappa_{d}}^{\kappa J}\right|^{2}+\left|\mathcal{M}_{\kappa_{d}}^{\kappa J}\right|^{2}\right)
$$

where $\mathcal{M}_{\kappa_{d}}^{\kappa J}$ and $\mathcal{E}_{\kappa_{d}}^{\kappa J}$ are respectively the general reduced matrix elements of the magnetic and electric transitions given in Appendix $\mathrm{B}$. The isovector $\mathcal{M}_{3}^{1} S_{0}$ transition is the only non-negligible M1 contribution at low energies and is furthermore non-vanishing only in the threshold domain. The result is in good agreement with experimental data,

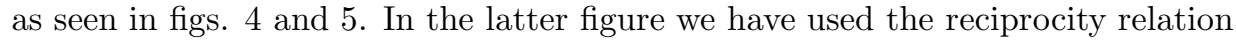

$$
\sigma_{\mathrm{n}}=\frac{3}{2}\left(\frac{\omega_{\gamma}}{k}\right)^{2} \sigma_{\gamma}
$$

for the inverse reaction $n p \rightarrow \gamma d$.

The relevant asymmetry observable for the reaction $\vec{\gamma} d \rightarrow n p$ may be expressed in terms of the deuteron photodisintegration helicity cross sections $\sigma_{\lambda}$ as

$$
\mathcal{A}_{\vec{\gamma}}=\frac{\sigma_{+}-\sigma_{-}}{\sigma_{+}+\sigma_{-}}=\frac{2 \operatorname{Re} \sum_{\kappa \kappa^{\prime} J} \sum_{\kappa_{d} \kappa_{d}^{\prime}}\left(\mathcal{M}_{\kappa_{d}}^{\kappa J} \widetilde{\mathcal{E}}_{\kappa_{d}^{\prime}}^{\kappa^{\prime} J}+\mathcal{E}_{\kappa_{d}}^{\kappa J *} \widetilde{\mathcal{M}}_{\kappa_{d}^{\prime}}^{\kappa^{\prime} J}\right)}{\sum_{\kappa \kappa_{d} J}\left(\left|\mathcal{M}_{\kappa_{d}}^{\kappa J}\right|^{2}+\left|\mathcal{E}_{\kappa_{d}}^{\kappa J}\right|^{2}+\left|\widetilde{\mathcal{E}}_{\kappa_{d}}^{\kappa J}\right|^{2}+\left|\widetilde{\mathcal{M}}_{\kappa_{d}}^{\kappa J}\right|^{2}\right)} .
$$

Here and later on, the parity admixed (weak) wavefunctions and amplitudes generated by the weak nuclear force will be tilded for clarity. Naturally, the absolute squares of the weak amplitudes in the denominator of eq. (14) may as well be ignored due to their diminutive size. Specializing to low partial waves they reduce to the results given e.g. in refs. [10] and [15]. The asymmetry may also be written following the notation fixed in fig. 11 as

$$
\mathcal{A}_{k}=\frac{2 \operatorname{Re}\left[\mathcal{M}_{1}^{*} \widetilde{\mathcal{E}}_{1}+\sum_{i=2}^{k} \mathcal{E}_{i}^{*} \widetilde{\mathcal{M}}_{i}\right]}{\left|\mathcal{M}_{1}\right|^{2}+\sum_{i=2}^{k}\left|\mathcal{E}_{i}\right|^{2}}
$$
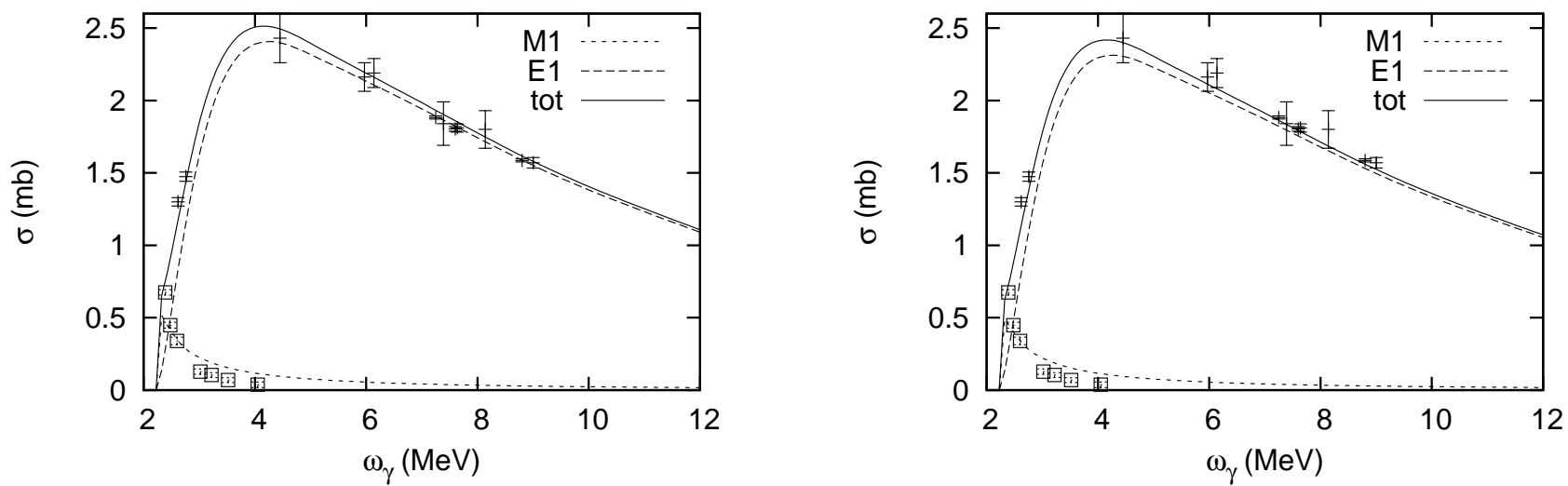

FIG. 4: The photodisintegration cross-sections of a deuteron for E1, M1, and their sum given by eq. (12). The Reid93 potential is employed in the left-hand side figure and the $\mathrm{A} v_{18}$ potential in the right-hand side figure. The data points ( $\square$ ) for M1 are taken from ref. [45] and $(+)$ for the total cross-section from ref. [46]. The $\omega_{\gamma}$ is the incident photon laboratory energy. 

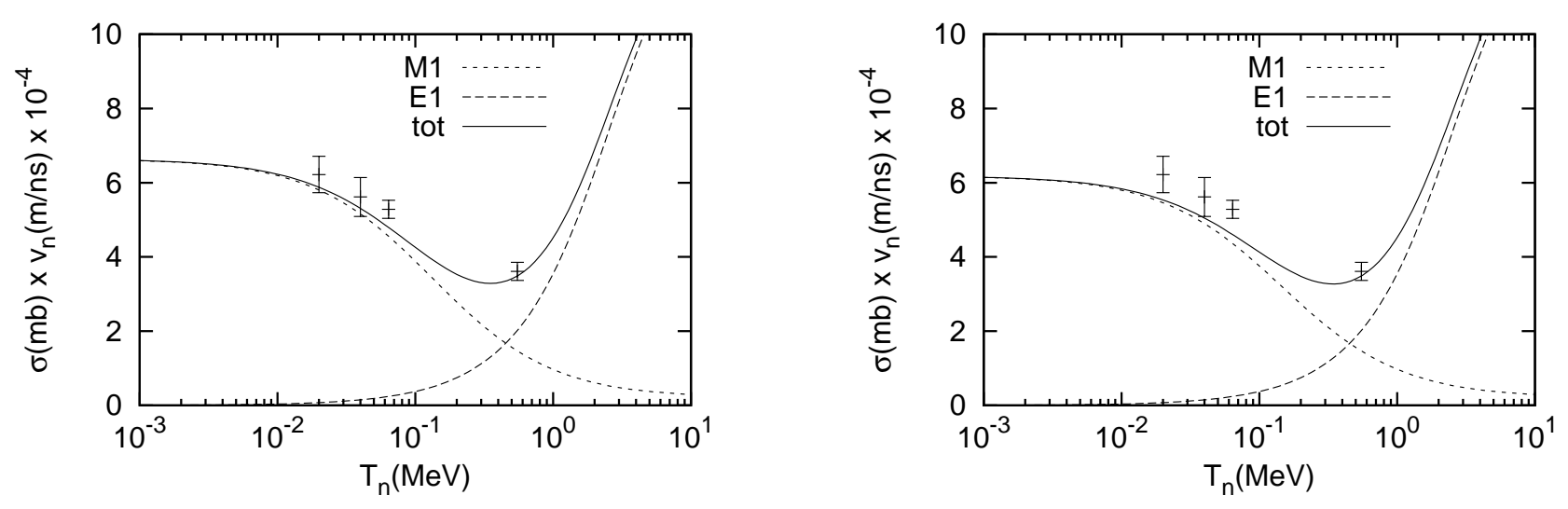

FIG. 5: The neutron capture cross-sections on a proton given by eq. (13) (the Reid93 potential is used in the left and the $\mathrm{A} v_{18}$ right). The data is from refs. 47, 48]. The lack of the meson-exchange currents (mainly the one-pion-exchange current correction) weakens the M1 of about $10 \%$ at low energies [49]. The neutron speed $v_{\mathrm{n}}$ (in the units of meters/nanosecond) and kinetic energy $T_{\mathrm{n}}$ are in the laboratory frame.

where the index $i$ denotes the final continuum channel. The total asymmetry $\mathcal{A}_{\vec{\gamma}}$ with $S$ and $P$ waves is achieved when $k=4$.

The magnetic dipole effect is dominant at the threshold where the asymmetry (15) reduces to a simple form $\mathcal{A}_{\vec{\gamma}}^{\text {thr. }}(k \rightarrow 0) \rightsquigarrow 2 \operatorname{Re}\left[\widetilde{\mathcal{E}}_{1} / \mathcal{M}_{1}\right]$, which is explicitly given by eqs. (B1) and (B2) as



It is important to note that eq. (16) arises from the spin changing PNC interaction, see fig. 1 and thus does not include the PNC pion exchange. Therefore, contributions from heavier vector meson exchanges (and possibly $\Delta$ ) are maximized. The low-energy limit (16) coincides with the photon polarization in the time-reversed reaction $n p \rightarrow \vec{\gamma} d$ for thermal neutrons.

The asymmetry from the radiative capture $\vec{n} p \rightarrow \gamma d$ of longitudinally polarized thermal neutrons in hydrogen is also calculated. The appropriate scattering wavefunctions depending on the spin magnetic quantum numbers $\left(m_{n}\right.$ and $m_{p}$ ) of the neutron and proton are obtained by expanding eq. (2). The wavefunctions (assuming the $z$-axis to be along the direction of $\boldsymbol{k}$ ) become

$$
\begin{aligned}
\psi_{m_{n} m_{p}}^{(-)}(k, \boldsymbol{r})= & \frac{\sqrt{4 \pi}}{k r} \sum_{\kappa^{\prime} \kappa} \sum_{J M_{S}} i^{L} \sqrt{2 L+1}\left\langle\frac{1}{2} m_{n} \frac{1}{2} m_{p} \mid S M_{S}\right\rangle \times \\
& \left\langle L 0 S M_{S} \mid J M_{S}\right\rangle \mathcal{U}_{\kappa \kappa^{\prime}}^{J(-)}(k, r) \mathcal{Y}_{J M_{S}^{\prime} S^{\prime}}^{L^{\prime}}(\hat{\boldsymbol{r}})(-1)^{T^{\prime}+1}\left|T^{\prime} 0\right\rangle .
\end{aligned}
$$

Since this is a time-reversed process to the deuteron disintegration, the amplitudes are of the form


tions $d \sigma_{m_{n}} / d \Omega$ with the neutron polarization $m_{n}$ and further in terms of the reduced matrix elements approximated for the thermal neutrons, reads $\mathcal{A}_{\vec{n}}(\theta)=\mathcal{A}_{\vec{n}} \cos \theta$ with

$$
\begin{aligned}
& \mathcal{A}_{\vec{n}}=\frac{d \sigma_{+\frac{1}{2}}-d \sigma_{-\frac{1}{2}}}{d \sigma_{+\frac{1}{2}}+d \sigma_{-\frac{1}{2}}} \approx \sqrt{2} \operatorname{Re}\left[\frac{\widetilde{\mathcal{E}}}{\mathcal{M}}\right]= \\
& \sqrt{2} \operatorname{Re}\left[\frac{i \int d r r \widetilde{\mathcal{D}}_{{ } P_{1}}(r)\left(\mathcal{U}_{3 S_{1}}^{(+)}(k, r)+\frac{1}{\sqrt{2}} \mathcal{U}_{3}^{(+)}(k, r)\right)-i \int d r r\left(\mathcal{D}_{{ }^{3} S_{1}}(r)+\frac{1}{\sqrt{2}} \mathcal{D}^{3}{ }_{D_{1}}(r)\right) \widetilde{\mathcal{U}}_{3}^{(+)}(k, r)}{\frac{\sqrt{3} \mu_{v}}{M} \int d r \mathcal{D}_{{ }} S_{1}(r) \mathcal{U}_{1}^{(+)}(k, r)}\right],
\end{aligned}
$$

where $\theta$ is the angle between the momenta of the incident neutron and emitted photon. Figure 6 presents this threshold result diagrammatically. Contrary to eq. (16), the $\mathcal{A}_{\vec{n}}$ is dominated by the spin conserving weak interaction and therefore the PNC one pion exchange prevails. Contrary to $\vec{\gamma} d \rightarrow n p$ in this reaction the $\Delta$-corrections are due to $\pi, \rho$, and $\omega$ exchanges. 


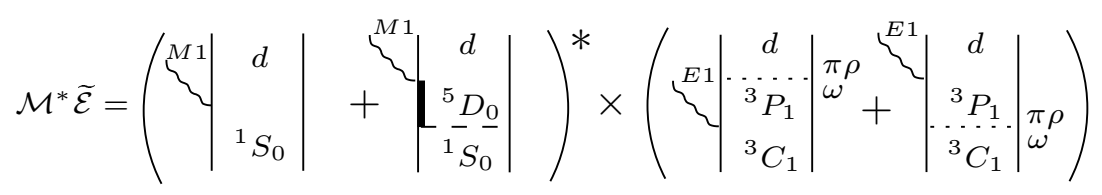

FIG. 6: Graphical representation for the PNC reaction $\vec{n} p \rightarrow \gamma d$ with thermal neutrons.
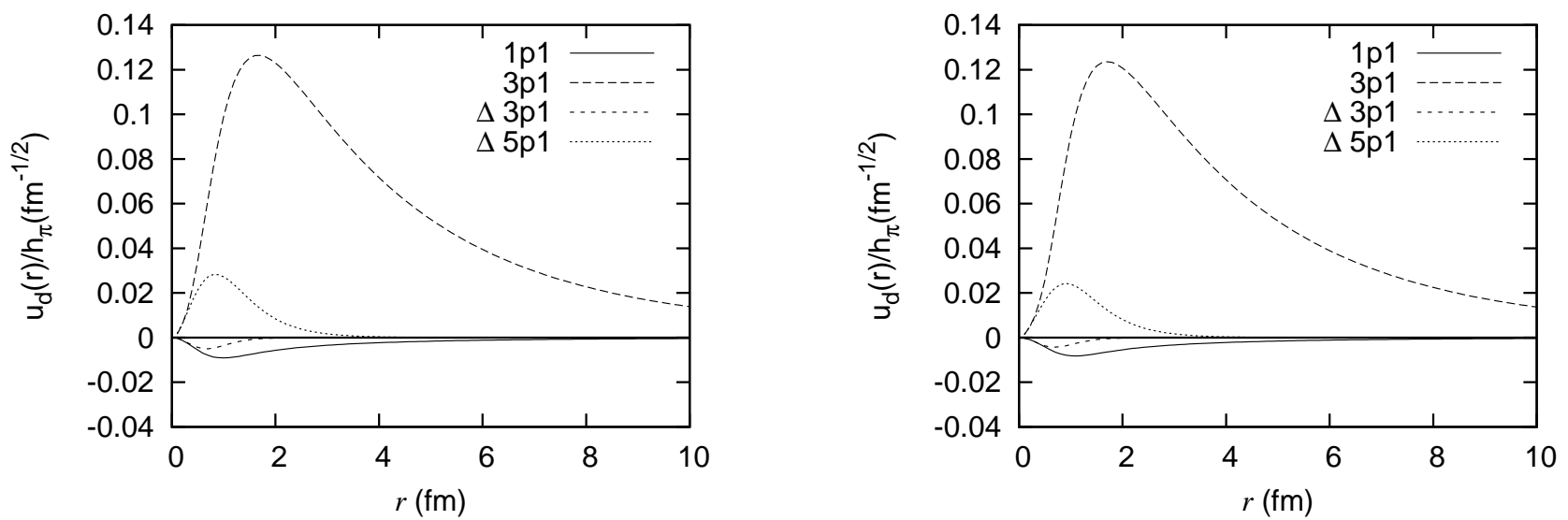

FIG. 7: The PNC deuteron components (divided by the DDH $h_{\pi}^{(1)}$ ) including the $\Delta$ with the DDH couplings and modified Yukawa functions. The Reid93 potential is used in the left panel and the $\mathrm{A} v_{18}$ in the right.

\section{RESULTS AND DISCUSSION}

Utilizing the different model complexes outlined in the previous section, we now proceed to calculate the asymmetries $\mathcal{A}_{\vec{\gamma}}$ for the reaction $\vec{\gamma} d \rightarrow n p$ as a function of photon laboratory energies varying from the deuteron breakup threshold to $10 \mathrm{MeV}$ and $\mathcal{A}_{\vec{n}}$ in the radiative capture of thermal neutrons $(25 \mathrm{meV}) \vec{n} p \rightarrow \gamma d$. It is also worth noticing that the photon polarization $\mathcal{P}_{\vec{\gamma}}$ of the reaction $n p \rightarrow \vec{\gamma} d$ with thermal neutrons is in principle the same as the observable of the time-reversed reaction at threshold $\mathcal{P}_{\vec{\gamma}} \approx \mathcal{A}_{\vec{\gamma}}\left(\omega_{\gamma}^{\text {th. }}\right)$. We also compare in some detail the asymmetries with and without the effects of the virtual $\Delta$-isobar.

In figs. 7 and 8 we show the PNC deuteron components relevant for the reactions discussed in this paper with and without form factors and also including $\Delta N$ admixture. Two phenomenological potentials are used, the updated Reid soft core 42] and Argonne $v_{18}$ [43] potentials. By far, the largest PNC component is ${ }^{3} P_{1}$, which mainly arises from pion exchange as seen in fig. 9] However, as was seen in sect. [I] and fig. 1] this does not contribute to PNC
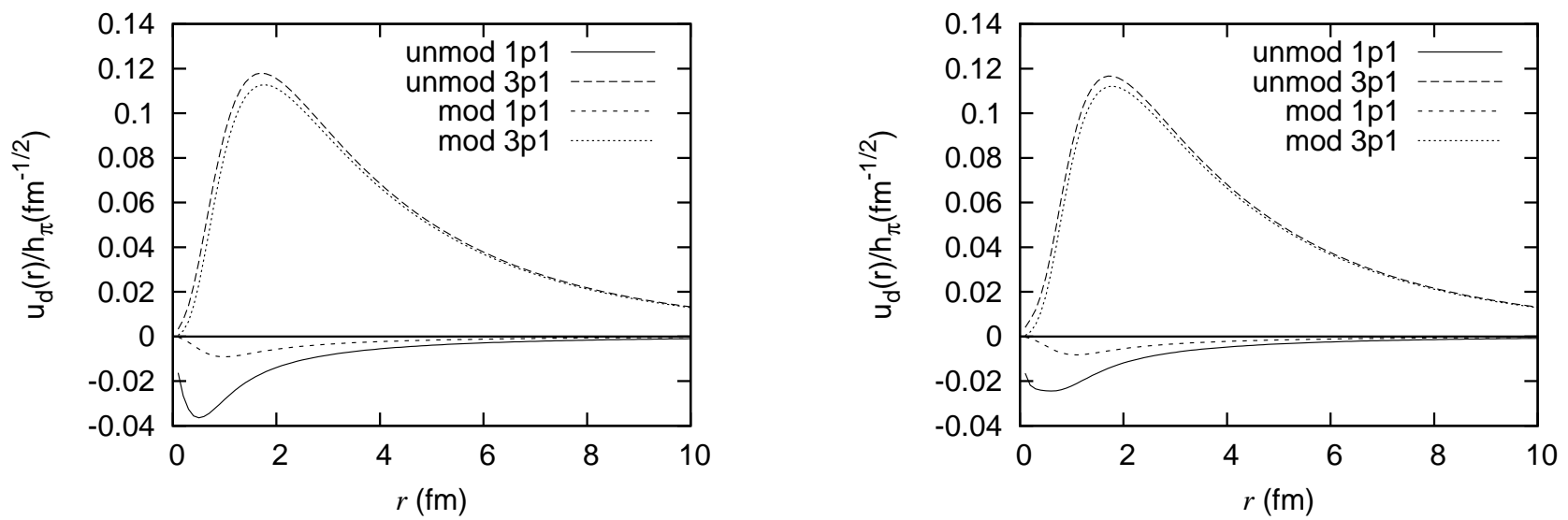

FIG. 8: The same as fig. 17, but without the $\Delta$. The components are compared using both unmodified and modified Yukawa functions. The result (unmodified) gained with $\mathrm{A} v_{18}$ potential is the same as in ref. [15]. 


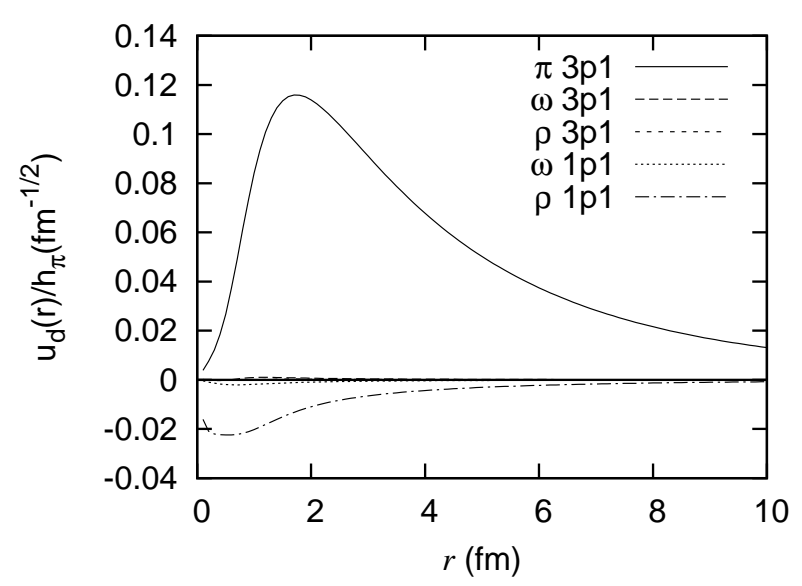

FIG. 9: Contributions of different mesons in different partial waves without the $\Delta$ using the DDH couplings, the A $v_{18}$ potential, and unmodified Yukawa functions.

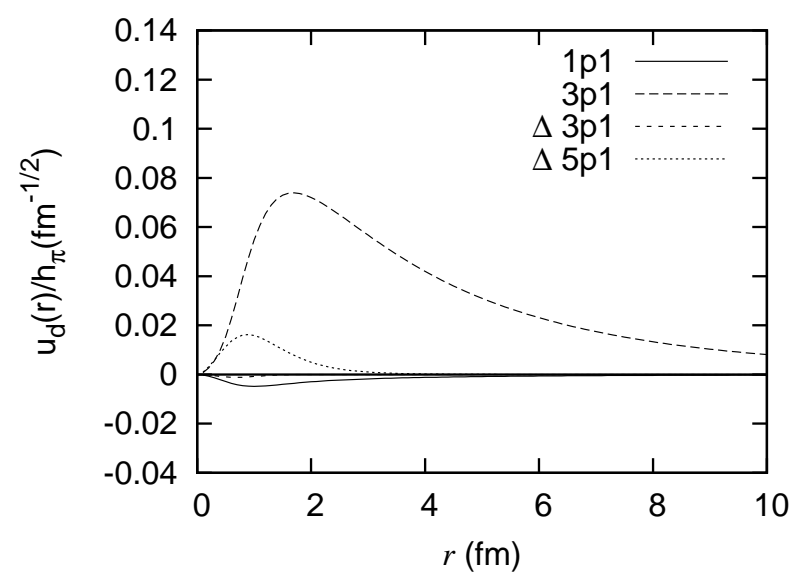

FIG. 10: The same as fig. 7 The components (divided by the DDH $h_{\pi}^{(1)}$ ) with the A $v_{18}$ potential and the FCDH couplings

photoabsorption into the lowest ${ }^{1} S_{0}$ partial wave and, consequently, is of minor importance at threshold. The ${ }^{1} P_{1}$ component arises from shorter ranged vector meson exchanges, notably $\rho$ and is smaller by an order of magnitude. The pion generated part ${ }^{3} P_{1}$ is relatively model independent (except for the weak pion coupling) whereas in ${ }^{1} P_{1}$ some short-range dependence can be seen, if the PNC potentials are not moderated by a form factor. Also it can be noted $\Delta N$ components can be of the same order as ${ }^{1} P_{1}$ although of shorter range. Therefore, it is plausible that their effect could be, in principle, appreciable and should be considered. Further, from fig. 9 it is obvious that with the standard DDH couplings $\omega$ exchange would be negligible and also $\rho$ in the pion dominated ${ }^{3} P_{1}$ component. Figure 10 shows another choice of weak couplings and the dependence on these is significant. The FCDH parametrization gives smaller PNC in comparison with fig. 7 Even though we have compromised to use the FCDH values, the other available weak coupling set with $\Delta$ by Desplanques [44] is alike and should give a similar result. Otherwise, if the weak couplings are the same, our PNC $N N$ wavefunctions are not in contradiction with those displayed in refs. [4, 9, 15].

We now consider the asymmetry $\mathcal{A}_{\vec{\gamma}}$, covering the photon energies from threshold to $10 \mathrm{MeV}$. Figure[11 illustrates separately the asymmetry of the "basic model" (by which we mean the commonly used model in the PNC calculations, which employs the DDH and strong interaction parameter values of table \without form factors) and the effect of the form factors and the $\Delta$ employing Reid93 and A $v_{18}$ potentials. In the case of the "basic model", the asymmetry is nearly $30 \%$ bigger with Reid93 at threshold. This difference is explained by the distinctly bigger $\mathrm{PNC}^{1} P_{1}$ component in the deuteron produced by Reid93 potential, as seen in fig. 8. However, with form factors in the PNC potentials the short-range differences are minimized and the results become graphically indistinguishable.

These points are further featured in tables II (last three columns) and III in the threshold limit $\omega_{\gamma}^{\text {lab }} \rightarrow 2.22592$ $\mathrm{MeV}$. Short-range correlation differences seem appreciable only in the transition from the PNC deuteron component 
TABLE II: Contributions of different mesons to the asymmetry $\mathcal{A}_{\vec{\gamma}}=\sum_{\alpha} \mathcal{A}_{\vec{\gamma}}^{\alpha}$, in units of $10^{-8}$, at threshold without $\Delta$-excitation using the unmodified Yukawa functions eq. (6). The threshold asymmetries are split into two pieces $\mathcal{A}_{\vec{\gamma}}^{\alpha}=\mathcal{A}_{d}^{3} \widetilde{P}_{0} \alpha+\mathcal{A}_{1}^{1} \widetilde{P}_{1} \alpha$, see eq. (16), where the indices characterize the E1 transition: subscripts the initial and superscripts the final states $(d$ for the PC part). The $\mathcal{A}_{\vec{\gamma}}^{\pi}=0$ in every case.

\begin{tabular}{|c||ccc|ccc|ccc|}
\hline \hline Model & $\mathcal{A}_{d}^{3} \widetilde{P}_{0} \rho$ & $\mathcal{A}_{1}^{1} \widetilde{P}_{1} \rho$ & $\mathcal{A}_{\vec{\gamma}}^{\rho}$ & $\mathcal{A}_{d}^{3} \widetilde{P}_{0} \omega$ & $\mathcal{A}_{1 \widetilde{P}_{1}}^{1} S_{0} \omega$ & $\mathcal{A}_{\vec{\gamma}}^{\omega}$ & $\mathcal{A}_{d}^{3} \widetilde{P}_{0}$ & $\mathcal{A}_{1 \widetilde{P}_{1}}^{1}$ & $\mathcal{A}_{\vec{\gamma}}$ \\
\hline $\mathrm{A} v_{18}$ \& DDH & -0.85 & 3.51 & 2.66 & -0.39 & 0.29 & -0.10 & -1.24 & 3.80 & 2.56 \\
Reid93 \& DDH & -0.84 & 4.10 & 3.26 & -0.38 & 0.38 & 0.00 & -1.22 & 4.48 & 3.26 \\
$\mathrm{~A} v_{18}$ \& FCDH & 0.41 & 1.17 & 1.58 & -1.00 & 0.76 & -0.24 & -0.59 & 1.93 & 1.34 \\
Reid93 \& FCDH & 0.40 & 1.36 & 1.76 & -0.99 & 0.99 & 0.00 & -0.59 & 2.35 & 1.76 \\
\hline \hline
\end{tabular}

TABLE III: Asymmetries $\mathcal{A}_{\vec{\gamma}}$ and $\mathcal{A}_{\vec{\gamma}}^{\Delta}$, in units of $10^{-8}$, respectively without and with $\Delta$-excitation using the modified Yukawa functions eq. (7).

\begin{tabular}{|c||ccc|ccc|}
\hline \hline Model & $\mathcal{A}_{d}^{3} \widetilde{P}_{0}$ & $\mathcal{A}_{1}^{1} \widetilde{P}_{1}$ & $\mathcal{A}_{\vec{\gamma}}$ & $\mathcal{A}_{d}^{3} \widetilde{P}_{0} \Delta$ & $\mathcal{A}_{1}^{1} \widetilde{P}_{1} \Delta$ & $\mathcal{A}_{\vec{\gamma}}^{\Delta}$ \\
\hline A $v_{18}$ \& DDH & -0.69 & 1.74 & 1.05 & -0.73 & 1.69 & 0.96 \\
Reid93 \& DDH & -0.69 & 1.83 & 1.14 & -0.73 & 1.77 & 1.04 \\
$\mathrm{~A} v_{18} \& \mathrm{FCDH}$ & -0.33 & 0.95 & 0.62 & -0.27 & 0.92 & 0.65 \\
Reid93 \& FCDH & -0.33 & 1.03 & 0.70 & -0.28 & 1.00 & 0.72 \\
\hline \hline
\end{tabular}

${ }^{1} \widetilde{P}_{1}$ to the ${ }^{1} S_{0}$ final state without form factors (table II), while with form factors the difference is hardly $10 \%$. The effect of the form factors themselves in PNC heavy meson exchanges is, however, a dramatic decrease to less than half of the original value of the asymmetry $\mathcal{A}_{\vec{\gamma}}$. In general the FCDH results are much smaller that DDH with and without form factors as seen in fig. 12 and Tables. The threshold result $\mathcal{A}_{\vec{\gamma}}=2.56 \times 10^{-8}$ gained with the "basic model" and the A $v_{18}$ potential argrees with most of the existing calculations and perfectly with those of using the same model [10, 15]. Even though the $N \Delta$ components in the deuteron appeared significant in fig. 7, their effect in $\mathcal{A}_{\vec{\gamma}}$ remains negligible at low energies being about $10 \%$ decrease for DDH at threshold and practically null for FCDH.

Figure 13 shows the importance of the $P$-wave continuum states above threshold as a cumulative behaviour of eq. (15). In the same figure are also shown the contributions of different mesons. As the photon energy increases, the asymmetry decreases steeply within the energies up to of about $1 \mathrm{MeV}$ above the threshold. This energy region is dominated by heavy meson exchanges, mainly the $\rho$. Increasing the energy further, the asymmetry quickly settles down near to zero continuing its gentle monotonic decline through zero somewhere between 3 and $4 \mathrm{MeV}$, after which the pion starts to take the dominance.

At low energies the pion contribution to $\mathcal{A}_{\vec{\gamma}}$ is small and, in our model and expressions, vanishes in the threshold limit (there is a negligible contribution higher order in $\boldsymbol{k}_{\gamma}$ arising from the spin current [4, 10]). This is not so for
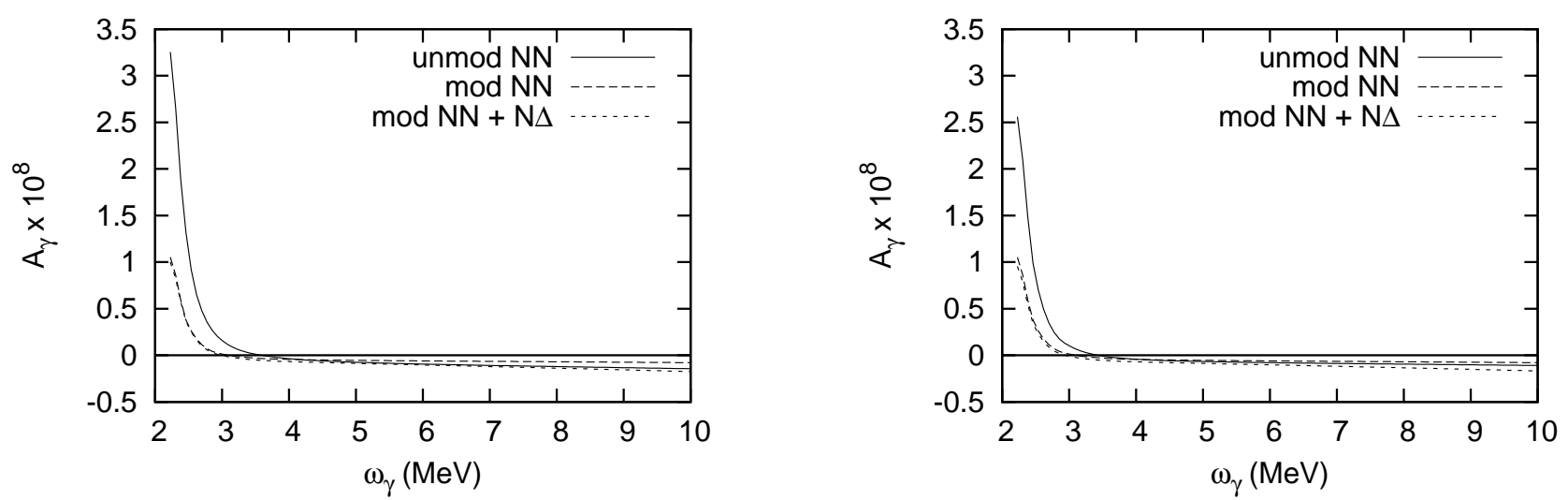

FIG. 11: The total asymmetry given by eq. (15) and the DDH couplings with unmodified and modified Yukawa functions without $(\mathrm{NN})$ and with the $\Delta$ excitation $(\mathrm{NN}+\mathrm{N} \Delta)$. The Reid93 left and the Av18 right. 


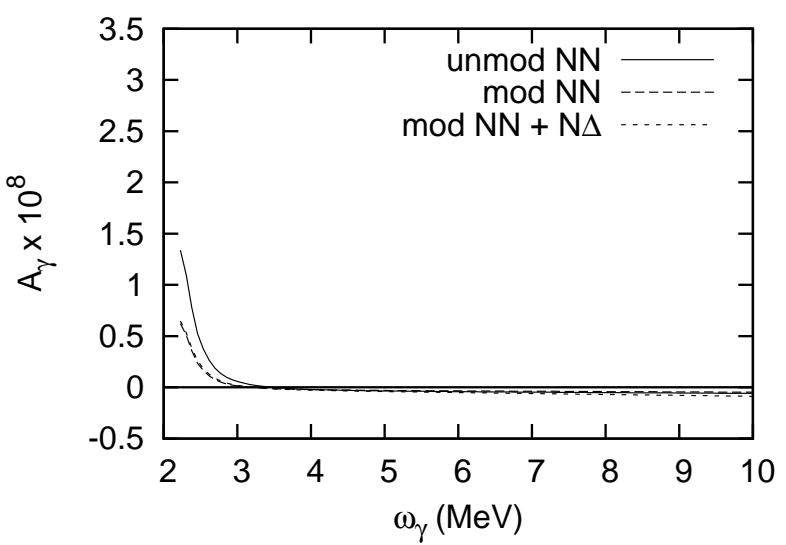

FIG. 12: The total asymmetry given by eq. (15), the Av $v_{18}$ potential, and the FCDH couplings with unmodified and modified Yukawa functions without $(\mathrm{NN})$ and with the $\Delta$ excitation $(\mathrm{NN}+\mathrm{N} \Delta)$.
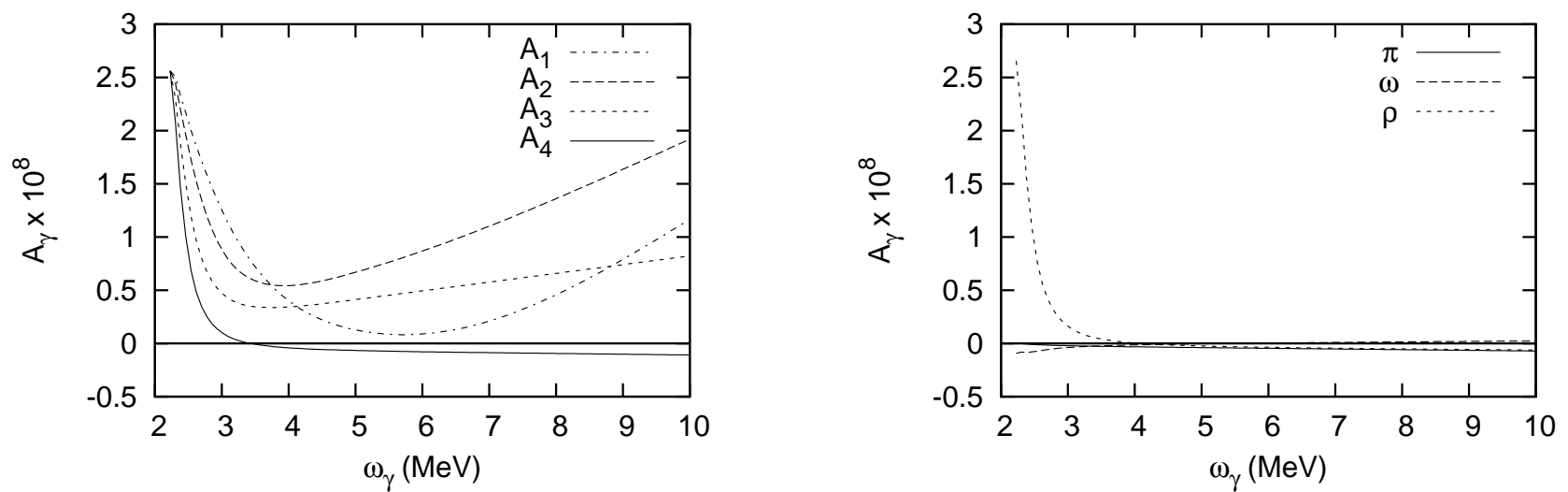

FIG. 13: Cumulative behaviour of the asymmetry eq. (15) in the left-hand side panel. Single meson $\pi, \omega$, and $\rho$ contributions to the asymmetry in the right-hand side panel. Both figures are plotted employing the DDH couplings, unmodified Yukawa functions and $\mathrm{A} v_{18}$ potential without $\Delta$ excitation.

radiative capture of longitudinally polarized neutrons, where it can participate in spin conserving PNC transitions ${ }^{3} S_{1}+{ }^{3} D_{1} \leftrightarrow{ }^{3} P_{1}$ as depicted in fig. [6 and expressed in eq. (18). As seen in table IV] contrary to $\mathcal{A}_{\vec{\gamma}}$, now the vector meson contributions are negligible. (It may still be of interest to note that now due to the spin conserving couplings $h_{\alpha}^{(1)} \omega$ is more important than $\rho$.) PNC in both bound states and continuum is about equal in magnitude and their contributions add constructively. Also in this case the value of $\mathcal{A}_{\vec{n}}=-5.39 \times 10^{-8}$ by the "basic model" and the A $v_{18}$ potential is in agreement with results of most authors and especially ref. 15] in which the wavefunctions of the same model are used. Due to the long range of the pion and low energies the form factor effect is only a $2-3 \%$ decrease in

TABLE IV: Contributions of different mesons to the $\mathcal{A}_{\vec{n}}=\sum_{\alpha} \mathcal{A}_{\vec{n}}^{\alpha}$, in units of $10^{-8}$, without $\Delta$-excitation using the unmodified Yukawa functions eq. (6). Furthermore, as in table II] the $\mathcal{A}_{\vec{n}}^{\alpha}$ is a sum of PNC scattering $\mathcal{A}_{3}^{d \alpha} \widetilde{P}_{1}$ and bound $\mathcal{A}_{3}^{3} \widetilde{P}_{1} \alpha$ contributions corresponding to eq. (18).

\begin{tabular}{|c||ccc|ccc|ccc|ccc|}
\hline \hline Model & $\mathcal{A}_{3 \tilde{P}_{1}}^{d \pi}$ & $\mathcal{A}_{3}^{3} \vec{P}_{1} \pi$ & $\mathcal{A}_{\vec{n}}^{\pi}$ & $\mathcal{A}_{3}^{d \rho} \widetilde{P}_{1}$ & $\mathcal{A}_{3}^{3} \vec{P}_{1} \rho$ & $\mathcal{A}_{\vec{n}}^{\rho}$ & $\mathcal{A}_{3}^{d \omega} \tilde{P}_{1}$ & $\mathcal{A}_{3}^{3} \vec{P}_{1} \omega$ & $\mathcal{A}_{\vec{n}}^{\omega}$ & $\mathcal{A}_{3 \widetilde{P}_{1}}^{d}$ & $\mathcal{A}_{3}^{3} \vec{P}_{1}$ & $\mathcal{A}_{\vec{n}}$ \\
\hline $\mathrm{A} v_{18}$ \& DDH & -2.85 & -2.51 & -5.36 & 0.00 & 0.00 & 0.00 & -0.01 & -0.02 & -0.03 & -2.86 & -2.53 & -5.39 \\
Reid93 \& DDH & -2.85 & -2.37 & -5.22 & 0.02 & 0.00 & 0.02 & -0.03 & -0.01 & -0.04 & -2.86 & -2.40 & -5.26 \\
$\mathrm{~A} v_{18}$ \& FCDH & -1.67 & -1.47 & -3.14 & 0.00 & 0.00 & 0.00 & -0.02 & -0.05 & -0.07 & -1.69 & -1.52 & -3.21 \\
Reid93 \& FCDH & -1.68 & -1.39 & -3.07 & 0.01 & 0.00 & 0.01 & -0.02 & -0.05 & -0.07 & -1.69 & -1.44 & -3.13 \\
\hline \hline
\end{tabular}


$\mathcal{A}_{\vec{n}}$. The $\Delta$ excitation contributes another $5 \%$ in both continuum and bound states, but these effects cancel off.

In summary, we have calculated the asymmetries $\mathcal{A}_{\vec{\gamma}}$ and $\mathcal{A}_{\vec{n}}$ in polarized photon absorption and in radiative capture of polarized neutrons close to threshold. The results are mutually complementary in the sense that $\mathcal{A}_{\vec{\gamma}}$ is dominated by vector meson exchange, while $\mathcal{A}_{\vec{n}}$ is pion dominated. The dominances are more than an order of magnitude with the minor effect being negligible. Also the $\Delta$ effects are small. In addition, very soon above threshold $\mathcal{A}_{\vec{\gamma}}$ becomes probably too small to be experimentally informative. In turn $\mathcal{A}_{\vec{n}}$ can give some limits to the weak pion coupling provided the error can be pushed below $10^{-8}$ as hoped for the NPDGamma experiment [35]. As for $\mathcal{A}_{\vec{\gamma}}$, being sensitive to short range effects, it could carry information on low energy constants of chiral perturbation theory.

\section{Acknowledgments}

T. M. P. would like to thank Vilho, Yrjö and Kalle Väisälä Foundation for the financial support of this work. This work was also supported by the Academy of Finland and DAAD researcher exchange grants 121892 and 139512 . We thank Institut für Kernphysik of Forschungszentrum Jülich for kind hospitality.

\section{Appendix A}

The parity conserving and nonconserving effective Hamiltonians (for PNC see [38]) for the $N N$ and $\Delta N$ vertices are given by

$$
\begin{gathered}
\mathcal{H}_{N N \rho}^{\mathrm{PC}}=g_{\rho} \bar{\psi}\left(\gamma^{\mu}+\frac{i \chi_{\rho}}{2 M} \sigma^{\mu \nu} q_{\nu}\right) \boldsymbol{\tau} \psi \cdot \boldsymbol{\rho}_{\mu}, \\
\mathcal{H}_{N N \rho}^{\mathrm{PNC}}=\bar{\psi}\left[h_{\rho}^{(0)} \boldsymbol{\tau} \cdot \boldsymbol{\rho}_{\mu}+h_{\rho}^{(1)} \rho_{\mu}^{0}+\frac{h_{\rho}^{(2)}}{2 \sqrt{6}}\left(3 \hat{\tau}_{0} \rho_{\mu}^{0}-\boldsymbol{\tau} \cdot \boldsymbol{\rho}_{\mu}\right)\right] \gamma^{\mu} \gamma_{5} \psi, \\
\mathcal{H}_{\Delta N \rho}^{\mathrm{PNC}}=h_{\rho}^{\star(0)} \bar{\psi} \Psi^{\mu i} \rho_{\mu i}+h_{\rho}^{\star(1)} \bar{\psi} \Psi^{\mu 0} \rho_{\mu 0}+\text { h.c. }, \\
\mathcal{H}_{\Delta N \rho}^{\mathrm{PC}}=i \frac{f_{\rho}^{\star}}{m_{\rho}} \bar{\psi} \gamma_{5} \gamma^{\nu} \boldsymbol{T} \Psi^{\mu} \cdot\left(\partial_{\nu} \boldsymbol{\rho}_{\mu}-\partial_{\mu} \boldsymbol{\rho}_{\nu}\right)+\text { h.c. }, \\
\mathcal{H}_{N N \omega}^{\mathrm{PC}}=g_{\omega} \bar{\psi}\left(\gamma^{\mu}+\frac{i \chi_{\omega}}{2 M} \sigma^{\mu \nu} q_{\nu}\right) \psi \omega_{\mu}, \\
\mathcal{H}_{N \Delta \omega}^{\mathrm{PNC}}=h_{\omega}^{\star(1)} \bar{\psi} \Psi^{\mu 0} \omega_{\mu}+\text { h.c. }, \\
\mathcal{H}_{N N \pi}^{\mathrm{PNC}}=\frac{h_{\pi}^{(1)}}{\sqrt{2}} \epsilon_{i j 0} \bar{\psi} \hat{\tau}_{i} \hat{\pi}_{j} \psi, \\
\mathcal{H}_{N \Delta \pi}^{\mathrm{PC}}=\frac{f_{\pi}^{\star}}{m_{\pi}} \bar{\psi} \Psi^{\mu i} \partial_{\mu} \hat{\pi}_{i}+\text { h.c.. }
\end{gathered}
$$

where $f_{\rho}^{\star} / m_{\rho}=g_{\rho}^{\star}\left(1+\chi_{\rho}\right) / 2 M$ and $\Psi^{\mu}$ is the Rarita-Schwinger vector-spinor field.

\section{Appendix B}

In this appendix, we give explicitly the reduced matrix elements of the Hamiltonian eq. (1) expressed in terms of Wigner coefficients. The factor $N=\sqrt{\alpha \pi \omega_{\gamma} / 2}$ in the reduced matrix elements (B1)-(B3) simplifies away in the asymmetry observables. The time-reversed adjoint electromagnetic amplitudes differ from the originals only by the 
phase factor of $-(-1)^{J}$, which cancels out in the absolute squares and interference. The reduced $N N$ matrix elements for the electric E1 transition are (with shorthand notations $\hat{J}=2 J+1, \hat{S}=2 S+1$, and $\hat{L}=2 L+1$ )

$$
\mathcal{E}_{\kappa_{d}}^{\kappa^{\prime} J}(k)=i N(-1)^{S^{\prime}} \sqrt{3 \hat{J} \hat{L}^{\prime} \hat{L}_{d}}\left\{\begin{array}{ccc}
L^{\prime} & J & S^{\prime} \\
1 & L_{d} & 1
\end{array}\right\}\left(\begin{array}{ccc}
L^{\prime} & 1 & L_{d} \\
0 & 0 & 0
\end{array}\right) \int d r r \mathcal{U}_{\kappa^{\prime} \kappa}^{J(+)}(k, r) \mathcal{D}_{\kappa_{d}}(r) \delta_{S^{\prime} S_{d}} .
$$

Similarly the reduced $N N$ matrix elements for the magnetic M1 transition are given by

$$
\begin{aligned}
\mathcal{N}_{\kappa_{d} \lambda}^{\kappa^{\prime} J}(k)= & \lambda N \frac{(-1)^{L^{\prime}}}{2 M} \sqrt{3 \hat{J}}\left[(-1)^{S^{\prime}} \sqrt{L_{d}\left(L_{d}+1\right) \hat{L}_{d}}\left\{\begin{array}{ccc}
L^{\prime} & J & S^{\prime} \\
1 & L_{d} & 1
\end{array}\right\} \delta_{S^{\prime} S_{d}}\right. \\
& \left.-(-1)^{S_{d}+J} \sqrt{6 \hat{S}^{\prime} \hat{S}_{d}}\left\{\begin{array}{ccc}
S^{\prime} & J & L^{\prime} \\
1 & S_{d} & 1
\end{array}\right\} \mu_{ \pm}\left[(-1)^{S_{d}} \pm(-1)^{S^{\prime}}\right]\left\{\begin{array}{ccc}
\frac{1}{2} & S^{\prime} & \frac{1}{2} \\
S_{d} & \frac{1}{2} & 1
\end{array}\right\}\right] \times \\
& \int d r \mathcal{U}_{\kappa^{\prime} \kappa}^{J(+)}(k, r) \mathcal{D}_{\kappa_{d}}(r) \delta_{L^{\prime} L_{d}} .
\end{aligned}
$$

with $\mu_{+}=\mu_{s}$ and $\mu_{-}=\mu_{v}$. The reduced matrix elements for the magnetic M1 $N \rightarrow \Delta$ transitions of particle 1 (for isospin transitions $0 \leftrightarrow 1$ and $1 \leftrightarrow 2$ )

$$
\begin{aligned}
\Delta_{\kappa_{d} \lambda}^{\kappa^{\prime} J}(k)= & \lambda N \frac{\mu^{\star}}{M}(-1)^{L^{\prime}+J} \sqrt{8 \hat{J} \hat{S}^{\prime} \hat{S}_{d}}\left\{\begin{array}{ccc}
S^{\prime} & J & L^{\prime} \\
1 & S_{d} & 1
\end{array}\right\}\left\{\begin{array}{ccc}
\xi^{\prime} & S^{\prime} & \frac{1}{2} \\
S_{d} & \xi & 1
\end{array}\right\} \times \\
& \int d r \mathcal{U}_{\kappa^{\prime} \kappa}^{J(+)}(k, r) \mathcal{D}_{\kappa_{d}}(r) \delta_{L^{\prime} L_{d}},
\end{aligned}
$$

where $\xi$ is the degree of freedom for spin: $1 / 2$ for a nucleon and $3 / 2$ for a Delta. In the transitions of particle 2 eq. (B3) has an additional phase of $-(-)^{S_{d}+S^{\prime}}$. Totally the M1 reduced matrix elements are given by $\mathcal{M}_{\kappa_{d} \lambda}^{\kappa^{\prime} J}(k)=$ $\mathcal{N}_{\kappa_{d} \lambda}^{\kappa^{\prime} J}(k)+\Delta_{\kappa_{d} \lambda}^{\kappa^{\prime} J}(k)$. For convenience we also define $\mathcal{M}_{\kappa_{d} \lambda}^{\kappa^{\prime} J}(k)=\lambda \mathcal{M}_{\kappa_{d}}^{\kappa^{\prime} J}(k)$. Despite the multiplication factor, the general results (B1) and (B2) reduce to the expressions of ref. [10] for the five lowest amplitudes.

[1] B. Desplanques, J. F. Donoghue, and B. R. Holstein, Ann. Phys. (N.Y.) 124, 449 (1980).

[2] S.-L. Zhu, C. M. Maekawa, B. R. Holstein, M. J. Ramsey-Musolf, and U. van Kolck, Nucl. Phys. A748, 435 (2005).

[3] M. J. Ramsey-Musolf and S. A. Page, Ann. Rev. Nucl. Part. Sci. 56, 1 (2006).

[4] R. Schiavilla, J. Carlson, and M. W. Paris, Phys. Rev. C70, 044007 (2004).

[5] R. Schiavilla, J. Carlson, and M. W. Paris, Phys. Rev. C67, 032501 (2003).

[6] H. C. Lee, Phys. Rev. Lett. 41, 843 (1978).

[7] T. Oka, Phys. Rev. D 27, 523 (1983).

[8] I. B. Khriplovich and R. V. Korkin, Nucl. Phys. A690, 610 (2001).

[9] M. Fujiwara and A. I. Titov, Phys. Rev. C69, 065503 (2004).

[10] C. P. Liu, C. H. Hyun, and B. Desplanques, Phys. Rev. C69, 065502 (2004).

[11] C. H. Hyun, J. W. Shin, and S.-i. Ando, Mod. Phys. Lett. A24, 827 (2009).

[12] C. P. Liu, Phys. Rev. C75, 065501 (2007).

[13] B. Desplanques, Nucl. Phys. A 242, 423 (1975).

[14] B. A. Craver, E. Fischbach, Y. E. Kim, and A. Tubis, Phys. Rev. D13, 1376 (1976).

[15] C. H. Hyun, S. J. Lee, J. Haidenbauer, and S. W. Hong, Eur. Phys. J. A24, 129 (2005).

[16] J. W. Shin, S. Ando, and C. H. Hyun, Phys. Rev. C 81, 055501 (2010).

[17] M. R. Schindler and R. P. Springer, Nucl. Phys. A846, 51 (2010).

[18] S.-i. Ando, C. H. Hyun, and J. W. Shin, Nucl. Phys. A844, 165 (2010).

[19] K. R. Lassey and B. H. J. McKellar, Phys. Rev. C11, 349 (1975).

[20] D. Tadic, Phys. Rev. 174, 1694 (1968).

[21] G. S. Danilov, Phys. Lett. B35, 579 (1971).

[22] K. R. Lassey and B. H. J. McKellar, Nucl. Phys. A260, 413 (1976).

[23] B. Desplanques, Phys. Lett. B512, 305 (2001).

[24] D. B. Kaplan, M. J. Savage, R. P. Springer, and M. B. Wise, Phys. Lett. B449, 1 (1999).

[25] M. J. Savage, Nucl. Phys. A695, 365 (2001).

[26] C. H. Hyun, T.-S. Park, and D.-P. Min, Phys. Lett. B516, 321 (2001).

[27] C. H. Hyun, S. Ando, and B. Desplanques, Eur. Phys. J. A32, 513 (2007). 
[28] C. H. Hyun, S. Ando, and B. Desplanques, Phys. Lett. B651, 257 (2007).

[29] E. D. Earle et al., Can. J. Phys. 66, 534 (1988).

[30] V. A. Knyazkov et al., Nucl. Phys. A417, 209 (1984).

[31] B. Wojtsekhowski and W. T. H. van Oers, JLab Letter-of-Intent 00-002 for PAC 17 (2000).

[32] E. Stiliaris, Eur. Phys. J. A24S2, 175 (2005).

[33] J. F. Cavaignac, B. Vignon, and R. Wilson, Phys. Lett. B67, 148 (1977).

[34] J. Alberi et al., Can. J. Phys. 66, 542 (1988).

[35] R. C. Gillis et al., J. Phys.: Conf. Ser. 239, 012012 (2010).

[36] M. J. Iqbal and J. A. Niskanen, Phys. Rev. C49, 355 (1994).

[37] J. A. Niskanen, T. M. Partanen, and M. J. Iqbal, Eur. Phys. J. A36, 295 (2008).

[38] G. B. Feldman, G. A. Crawford, J. Dubach, and B. R. Holstein, Phys. Rev. C43, 863 (1991).

[39] A. J. F. Siegert, Phys. Rev. 52, 787 (1937).

[40] T. Ericson and W. Weise, Pions and Nuclei (Clarendon Press, Oxford, 1988).

[41] G. E. Brown and W. Weise, Phys. Rept. 22, 279 (1975).

[42] V. G. J. Stoks, R. A. M. Klomp, C. P. F. Terheggen, and J. J. de Swart, Phys. Rev. C49, 2950 (1994).

[43] R. B. Wiringa, V. G. J. Stoks, and R. Schiavilla, Phys. Rev. C51, 38 (1995).

[44] B. Desplanques, Nucl. Phys. A335, 147 (1980).

[45] W. Tornow et al., Phys. Lett. B574, 8 (2003).

[46] H. Arenhövel and M. Sanzone, Few-Body Syst. Suppl. 3 (1991).

[47] T. S. Suzuki, Y. Nagai, T. Shima, T. Kikuchi, H. Sato, T. Kii, and M. Igashira, Astrophys. Lett. 439, L59 (1995).

[48] Y. Nagai, T. S. Suzuki, T. Kikuchi, T. Shima, T. Kii, H. Sato, and M. Igashira, Phys. Rev. C56, 3173 (1997).

[49] D. O. Riska and G. E. Brown, Phys. Lett. 38B, 193 (1972). 